\title{
NGHIÊN CỬU ĐỀ XUẤT GIẢI PHÁP NÂNG CAO Độ CHÍNH XÁC CỦA CôNG TÁC PHÂN LOẠI ẢNH KHU VỰC CÓ LỚP PHỦ HỖN HỢP - PHẦN CƠ SỞ KHOA HỌC
}

\author{
TS. PHAM MINH HẢI \\ Viện Khoa học Đo đạc và Bản đồ
}

\section{Tóm tắt:}

Ngày nay, các ứng dụng ảnh vệ tinh phục vụ cho các mục đích nghiên cứu, thí nghiệm hay ứng dụng phục vụ đời sống xã hội nói chung và lĩnh vực Tài nguyên Môi trường nói riêng ngày càng trở nên phổ biến. Quá trình chiết tách thông tin được sử dụng phổ biến là các phương pháp phân loại ảnh có kiểm định và không có kiểm định. Phân loại có kiểm định là một phương pháp xác suất có khả năng sắp xếp những điểm ảnh do người sử dụng định nghĩa thành những lớp khác nhau. Tuy nhiên, khi thực hiện phân loại ảnh có kiểm định ở khu vực có bề mặt lớp phủ hỗn hợp, độ chính xác của kết quả phân loại ảnh không cao do các kết quả phân loại ảnh bị ảnh hưởng bởi vấn đề nhiễu điểm ảnh. Nhiễu điểm ảnh là hiện tượng xảy ra khi một điểm ảnh có giá trị điểm ảnh thuộc lớp A nhưng trong kết quả phân loại ảnh thì điểm ảnh đó sẽ được phân loại vào lớp khác ngoài lớp A. Trong các ứng dụng sử dụng tư liệu ảnh vệ tinh độ phân giải vì̛a và nhỏ, bề mặt đất hỗn hợp bao gồm nhiều đối tượng ảnh, công tác xử lý vấn đề nhiễu điểm ảnh bằng việc can thiệp vào các thành phần đất, nước, thực vật trên mỗi điểm ảnh nhằm cải thiện độ chính xác của kết quả phân loại là có tính cấp thiết cao. Trong phạm vi bài báo này, nhóm nghiên cứu sẽ giới thiệu cơ sở khoa học của giải pháp nâng cao độ chính xác của công tác phân loại ảnh khu vực có lớp phủ hỗn hợp. Phần thực nghiệm sẽ được trình bày trong số tới của Tạp chí Khoa học Đo đạc và Bản đồ.

\section{Lò̀i mở đầu}

Trong lĩnh vực giám sát Tài nguyên và Môi trường, phương pháp phân loại ảnh được sử dụng để khai thác dữ liệu ảnh vệ tinh. Quá trình tách thông tin từ ảnh vệ tinh có thể được thực hiện bằng các phương pháp phân loại ảnh. Hai phương pháp phân loại ảnh thông dụng hiện nay là phương pháp phân loại không kiểm định và phương pháp phân loại có kiểm định. Trong phạm vi nghiên cứu này, đối tượng nghiên cứu để nâng cao độ chính xác là phương pháp phân loại có kiểm định.

Bề mặt đất được ghi lại bởi các điểm ảnh, mỗi điểm ảnh thường chứa nhiều hơn

Ngày nhận bài: 24/5/2016 một loại lớp phủ. Nhiễu điểm ảnh là hiện tượng xảy ra khi một điểm ảnh có giá trị điểm ảnh thuộc lớp $A$ nhưng trong kết quả phân loại ảnh thì điểm ảnh đó sẽ được phân loại vào lớp khác ngoài lớp $A$. Ba đối tượng đặc trưng được mô tả tồn tại trong mỗi điểm ảnh đó là: Nước-Đất-Thực vật. Mỗi đối tượng này sẽ chiếm tỷ lệ nhất định trong mối điểm ảnh. Ví dụ, nếu một điểm ảnh có tỷ lệ Nước: $50 \%$, Đất: 30\%, Thực vật: 20\% thì điểm ảnh này sẽ thuộc lớp Nước do đối tượng nước chiếm tỷ lệ cao nhất trong điểm ảnh. Độ chính xác công tác phân loại ảnh bị chi phối bởi hiện tượng nhiễu điểm ảnh sau phân loại ảnh. Tuy nhiên, cho tới nay tại nước ta các nghiên cứu về giảm nhiễu điểm

Ngày chấp nhận đăng: 06/6/2016 
ảnh chưa quan tâm nhiều, phát triển giải pháp nâng cao độ chính xác kết quả phân loại ảnh có tính cấp thiết cao, nâng cao hiệu quả khai thác dữ liệu viễn thám. Trên cơ' sở' đánh giá tình hình thực tế, đề tài: "Nghiên cứu giải pháp kỹ thuật nâng cao độ chính xác của công tác phân loại ảnh khu vực có lớp phủ hỗn hợp" đã được thực hiện tại Viện Khoa học Đo đạc và Bản đồ, Bộ Tài nguyên và Môi trường.

\section{Khái niệm về hiện tượng nhiễu điểm ảnh}

Nhiễu điểm ảnh được định nghĩa là kết quả của sự hỗn hợp các đối tượng, thành phần trong một điểm ảnh, và độc lập với độ phân giải [5]. Nhiễu điểm ảnh thường xảy ra do kích thước các đối tượng và sự vật cần phân loại thường nhỏ hơn kích thước 1 điểm ảnh. Theo [8], có 3 trường hợp phổ biến về hiện tượng nhiễu điểm ảnh. Đó là:

- Nhiễu giữa các đối tượng trên 1 điểm ảnh như cửa, cây cối, ao, hồ .v.v...

- Nhiễu giữa đối tượng nằm trên vùng chuyển tiếp của các đối tượng trên 1 điểm ảnh như khu vực có nhiều cây cối .v.v...

- Nhiễu giữa các đối tượng có cấu trúc hình tuyến như cầu cống, đường xá .v.v...

Có 2 phương pháp chính trong giảm nhiễu điểm ảnh là phương pháp phân loại ảnh và phương pháp hình học. Với phương pháp phân loại ảnh, độ chính xác của sản phẩm phân loại phụ thuộc chính vào điều kiện lấy mẫu phân loại. Độ chính xác của sản phẩm được nâng cao sau nhiều lần thực nghiệm. Đây là phương pháp truyền thống được sử dụng nhiều nhất trong phân loại ảnh. Phương pháp hình học là phương pháp chiết tách thông tin các đối tượng trên ảnh, như nhà cửa, cây cối dựa trên hình dạng hay sự phân bố có quy luật trong không gian của chúng. Trong nghiên cứu của [22], phương pháp hình học được áp dụng để chiết tách thông tin về cây trồng trên ảnh viễn thám. Một cửa sổ di chuyển được sử dụng để thu thập mẫu phân loại về sự phân bố đối tượng cây thân gỗ và vùng đệm xung quanh. Việc lặp lại theo quy luật phân bố của đối tượng được thu thập đem đến kết quả hình học của mẫu được thu thập.

Các kết quả các nghiên cứu ở trên đã góp phần nâng cao độ chính xác kết quả phân loại ảnh, nhưng phần lớn các nghiên cứu này chỉ chú trọng vào nâng cao độ chính xác của kết quả phân loại ảnh dựa trên cơ sở tăng độ chính xác công tác lấy mẫu đối với công tác phân loại có kiểm định hay dựa vào hình dáng đối. Tuy nhiên để đạt được kết quả có độ chính xác cao, quá trình xử lý ảnh lặp đi lặp lại đòi hỏi thời gian. Hơn nữa, độ chính xác của kết quả phân loại thực hiện bởi những phương pháp này cũng phụ thuộc nhiều vào ý thức chủ quan của người thực hiện. Do vậy, một giải pháp nâng cao độ chính xác công tác phân loại ảnh có mức độ tự động hóa cao giảm sự can thiệp của con người đóng vai trò cấp thiết cao, nâng cao khả năng khai thác dữ liệu viễn thám phục vụ các nhu cầu của người sử dụng công nghệ viễn thám.

3. Cơ sở khoa học phương pháp giảm nhiễu điểm ảnh bằng phân loại theo giá trị phổ sử dụng 3 đối tượng đất, nước, thực vật

\subsection{Chỉ số thực vật PVI}

PVI (Perpendicular Vegetation Index) là chỉ số thực vật được Richardson và Wiegand (1977) đề xuất. Khi tính toán chỉ số $\mathrm{PVI}$, đường Đường đất (Soil line) đóng một vai trò quan trọng. $\mathrm{PVI}$ được thể hiện trong hệ tọa độ 2 chiều giữa trục tung là kênh cận hồng ngoại và trục hoành là kênh đỏ. Trong hệ tọa độ này, biểu đồ phân tán phổ của khu vực nghiên cứu được thể hiện và dựa vào đây chúng ta có thể phân biệt được khu vực đất ẩm và đất trống. (xem hình 1)

Trên hình 1 thể hiện đường đất, trên 
đường này phổ của khu vực có đất khô nằm ở trên phía tay phải của đường và ngược lại. Khi lượng thực vật tăng, sự phản xạ kênh cận hồng ngoại tăng và kênh đỏ giảm, khi đó sự phân bố phổ sẽ dồn vào phía trên bên dưới trái của đường đất. Khi lượng thực vật giảm, yếu tố đất của nền ảnh sẽ tăng do vậy các phản xạ đất cũng tăng và khi đó đường đất sẽ có xu hướng đi theo chiều ngang. Sự ảnh hưởng của đất sẽ được hạn chế tối thiểu để tính toán thực vật bằng cách tính khoảng cách từ đường đất đến điểm quan sát trong sơ đồ phân bố phổ $\mathrm{PVI}$.

\subsection{Cơ sở khoa học của nghiên cứu}

Kế thừa phương pháp tính toán chỉ số thực vật PVI, nhóm nghiên cứu tiếp tục phát triển tính toán tỷ lệ thành phần đất, nước thực vật từ chỉ số PVI. Từ hình 1 , chúng ta thấy nếu biểu diễn các điểm ảnh trong mối tương quan kênh đỏ và cận hồng ngoại thì đối tượng thực vật sẽ được phân bố ở phía trên đường đất, với các đối tượng đất ẩm có nước sẽ phân bố ở phía bên trái đường đất, và cuối cùng với các đối tượng đất sẽ phân bố ở phía bên trái đường đất. Khi nối các điểm ảnh ở vị trí cực đại $x_{\max }, y_{\max }, x_{\min }$, $y_{\min }$ sẽ tạo thành một hình tam giác. Miền tam giác này sẽ chứa tất cả các điểm ảnh

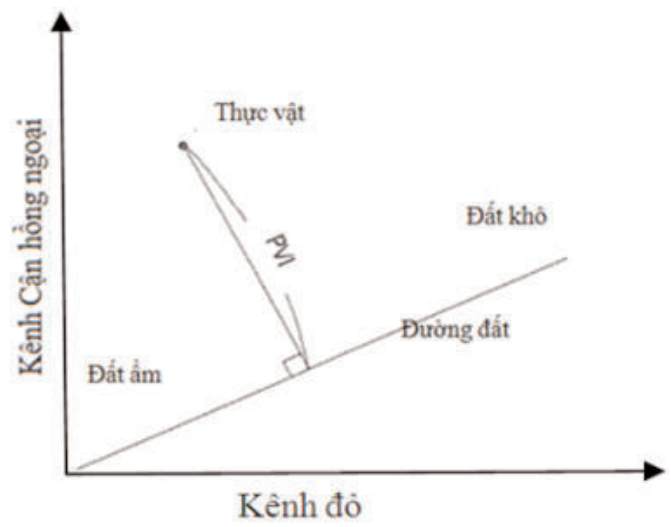

Hình 1: Minh họa chỉ số PVI trên ảnh, đây là miền giới hạn vùng tính toán. Trong nghiên cứu này các điểm ảnh ở vị trí Thực vật $\left(x, y_{\max }\right)$, Nước $\left(x_{\operatorname{mix}}, y_{\min }\right)$, Đất $\left(x_{\max }, y\right)$ là các điểm ảnh có giá trị phổ được coi như giá trị phổ thuần khiết (endmember), giá trị phổ chưa bị trộn lẫn.

Việc xác định được các endmember này trên ảnh có nghĩa xác định được không gian giới hạn công tác xử lý ảnh. Tất cả các điểm ảnh trên ảnh sẽ nằm trong miền tam giác tạo bởi 3 đỉnh đất, nước, thực vật trên. Miền tính toán sẽ bao gồm toàn ảnh do vậy một con chạy $i$ sẽ được thiết kế để chạy qua tất cả các điểm ảnh trên ảnh. Việc tính tỷ lệ thành phần đất, nước, thực vật của mối điểm ảnh trên phạm vi toàn ảnh sẽ được mô tả ở các phần sau.

\subsection{Xác định vị trí 3 đỉnh của tam giác phổ đất, nước, thực vật}

Sau khi đã khái niệm được miền tam giác tạo bởi 3 đỉnh đất, nước, thực vật (xem hình 2). Nhóm nghiên cứu thiết lập một phương trình chiết tách các giá trị phổ thực của đất, nước, thực vật như sau:

$f_{\text {endmember }}=f$ (endmembera, endmemberb,endmemberc)

Ở đó: $\mathrm{f}_{\text {endmember }}$ = hàm số tổng chiết

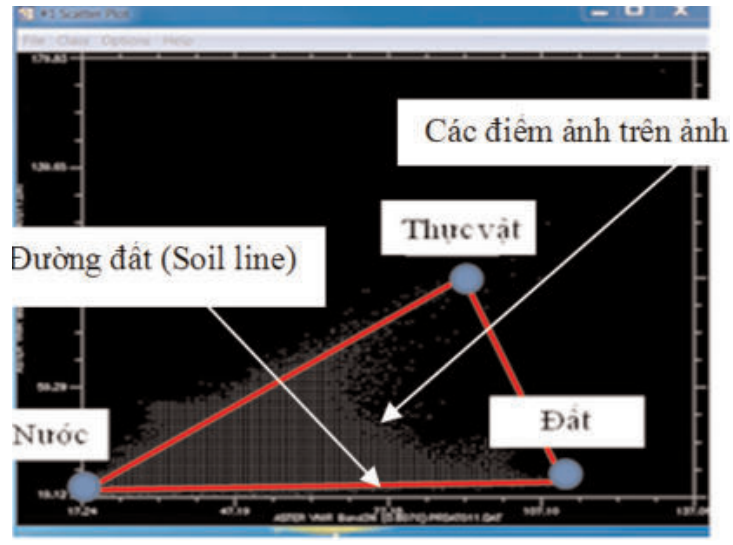

Hình 2: Phương pháp luận tính toán tỷ lệ thành phần đất, nước, thực vật phát triển từ chỉ số PVI 
tách 3 endmember đất, nước, thực vật (xem hinh 3, 4, 5)

- endmembera = hàm số chiết tách

endmember nước $=a\left(x_{\operatorname{mix}}, y_{\min }\right)$

- endmemberb = hàm số chiết tách

endmember thực vật $=\mathrm{b}\left(\mathrm{x}, \mathrm{y}_{\max }\right)$

- endmemberc = hàm số chiết tách

endmember đất $=\mathrm{c}\left(\mathrm{x}_{\max }, \mathrm{y}\right)$

Với giá trị enmember nước

endmember nước $=a\left(x_{\text {mix }}, y_{\min }\right)$

Với giá trị enmember thực vật

endmember thực vật $=b\left(x, y_{\max }\right)$

Với giá trị enmember đất

endmember đất $=c\left(x_{\max }, y\right)$

\subsection{Xác định tỷ lệ đất, nước, thực vật}

Giả sử ta có điểm quan trắc $\mathrm{P}\left(\mathrm{x}_{\mathrm{p}}, \mathrm{y}_{\mathrm{p}}\right)$ bất kỳ trong tam giác phổ tạo bởi 3 đỉnh Thực vật, Nước, Đất. Từ $\mathrm{P}$ hạ đường vuông góc xuống các cạnh của hình tam giác (xem hình 6). Trong mỗi điểm ảnh, 3 đối tượng đặc trưng được thể hiện là đất, nước, thực vật. Độ dài các đường thẳng $\mathrm{PV}, \mathrm{PS}, \mathrm{PW}$ thể hiện tỷ lệ của thực vật, đất, nước trên mỗi điểm ảnh. Nhóm nghiên cứu giả định

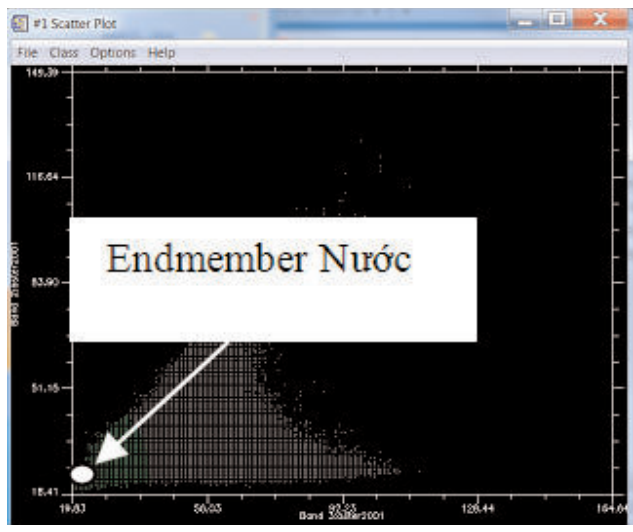

Hình 3: Minh họa cách xác định endmember nước nếu $P V>P S$ và $P W$ thì thành phần thực vật sẽ chiếm tỷ trọng chủ yếu trong điểm ảnh, do đó điểm ảnh đó sẽ được phân tới lớp "Thực vật". Tương tự như vậy tính toán cho trường hợp của $\mathrm{PS}$ và $\mathrm{PW}$.

Với mỗi điểm quan trắc $P\left(x_{p}, y_{p}\right)$, chúng ta đều có thể tính toán được tỷ lệ của mỗi yếu tố đất, nước, thực vật qua các bước sau:

Bước 1: Tính toán giá trị cực đại, cực tiểu trên 2 kênh Đỏ và Cận hồng ngoại để xác định $V\left(x_{v}, y_{v}\right), W\left(x_{w}, y_{w}\right), S\left(x_{s}, y_{s}\right)$.

Bước 2: Giới hạn phạm vi tính toán trong tam giác phổ:

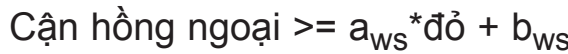

Cận hồng ngoại $<=a_{v s}{ }^{*}$ đỏ $+b_{v s}$

Cận hồng ngoại $>=a_{v w}{ }^{*}$ đỏ $+b_{v w}$

Bước 3: Tính toán 6 tham số $a_{w s}, b_{w s}$, $a_{v s}, b_{v s}, a_{v w}, b_{v w}$.

Tính toán tham số $a_{w s}, b_{w s}$ của đường thẳng $\mathrm{y}_{\mathrm{v}}=\mathrm{a}_{\mathrm{ws}} * \mathrm{x}_{\mathrm{v}}+\mathrm{b}_{\mathrm{ws}}$ bằng công thức sau: $\quad a_{w s}=\frac{y_{w}-y_{s}}{x_{w}-x_{s}}$

$$
b_{w s}=y_{w a}-a_{w a}{ }^{*} x_{w}
$$

Tương tự công thức 6 và 7 tính toán cho $a_{v s}, b_{v s}, a_{v w}, b_{v w}$.

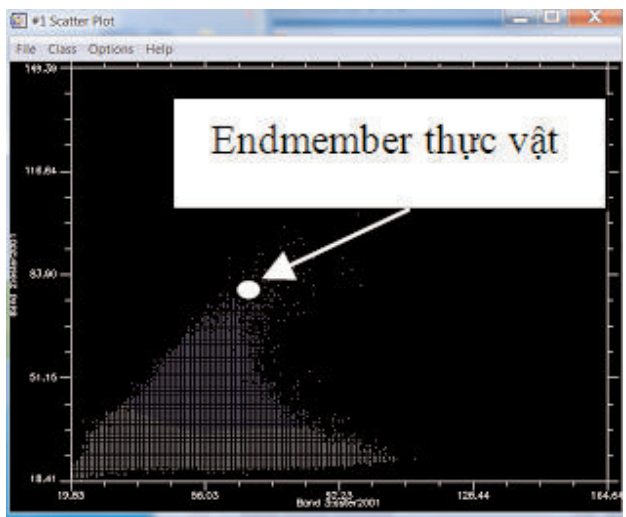

Hình 4: Minh họa cách xác định endmember thực vật 
Bước 4: Tính toán tham số $a_{p}, b_{p}$ của đường thẳng $\mathrm{y}_{\mathrm{p}}=\mathrm{a}_{\mathrm{p}} * \mathrm{x}_{\mathrm{p}}+\mathrm{b}_{\mathrm{p}}$

$$
\begin{aligned}
& a_{p}=\frac{-1}{a_{w s}} \\
& b_{p}=y_{p}-a_{p}{ }^{*} x_{p}
\end{aligned}
$$

Bước 5: Tính tọa độ điểm $V\left(x_{v}, y_{v}\right)$

$$
\begin{aligned}
& x_{v}=\frac{\left(b_{p}-b_{w s}\right)}{\left(a_{p}-a_{w s}\right)} \\
& \mathrm{yv}=\mathrm{x}_{\mathrm{v}}{ }^{*} \mathrm{a}_{\mathrm{ws}}+\mathrm{b}_{\mathrm{w}}
\end{aligned}
$$

Tương tự tính toán cho tọa độ

$W\left(x_{w}, y_{w}\right), S\left(x_{s}, y_{s}\right)$.

Bước 6: Tính độ dài PV, PW, PS

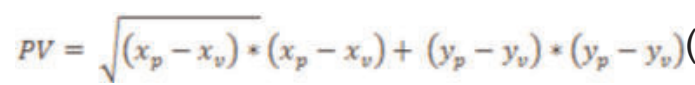

Tương tự tính toán cho độ dài PW, PS.

Bước 7: So sánh độ dài 3 cạnh PV, PW, PS đã tính.

Nếu $P V>P W$ và $P S$ thì điểm ảnh sẽ có tỷ lệ thực vật lớn nhất.

Nếu $P W>P V$ và $P S$ thì điểm ảnh sẽ có tỷ lệ nước lớn nhất.

Nếu $P S>P W$ và $P V$ thì điểm ảnh sẽ có tỷ lệ nước lớn nhất.

Việc tính toán các giá trị PV, PW, PS trên toàn ảnh sẽ cung cấp kết quả là 3 ảnh chỉ số đất, nước, thực vật. Mỗi ảnh chỉ số thực

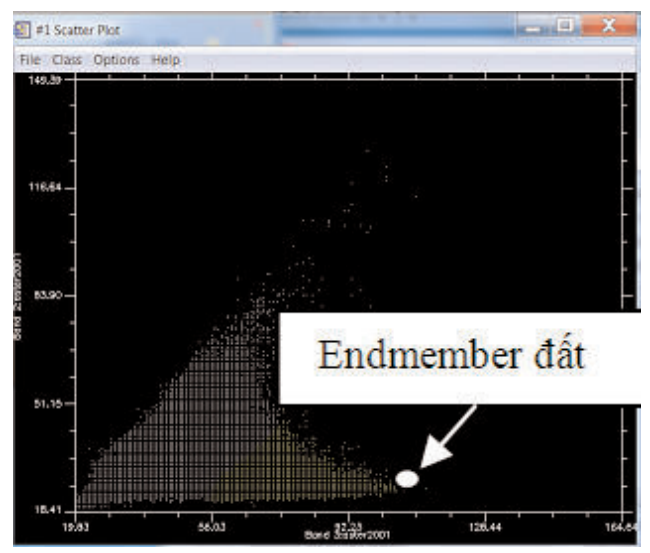

Hình 5: Minh họa cách xác định endmember đất vật có giá trị $\mathrm{DN}$ biểu diễn ở thang độ xám 8 bit. Trong đó, trên ảnh chỉ giá trị của các điểm ảnh càng lớn thì đối tượng mô tả (đất, nước, thực vật) sẽ được tương phản càng rõ nét trên ảnh.

\section{Kết luận}

Nghiên cứu đã góp phần giải quyết những vấn đề liên quan đến giảm nhiễu của các đối tượng trên ảnh đồng thời nâng cao chính xác kết quả phân loại ảnh. Cơ sở khoa học của giải pháp của giải pháp nâng cao độ chính xác của công tác phân loại ảnh khu vực có lớp phủ hỗn hợp đã được phát triển thành công. Việc sử dụng sự tương quan kênh đỏ và cận hồng ngoại trong tính toán tỷ lệ đất, nước, thực vật trong mỗi điểm ảnh sẽ cung cấp kết quả là 3 ảnh chỉ số đất, nước, thực vật. Ở đó, ảnh chỉ số nước và thực vật được thực nghiệm có độ chính xác về vị trí địa vật và độ tương phản tốt hơn ảnh chỉ số thực vật NDVI và chỉ số nước NWDI truyền thống. Việc phát triển được cơ sở khoa học của giải pháp nâng cao độ chính xác của công tác phân loại ảnh khu vực có lớp phủ hỗn hợp sẽ góp phần làm nâng cao độ chính xác kết quả phân loại ảnh đồng thời làm đơn giản hóa công tác phân loại ảnh viễn thám ít phụ thuộc vào chủ quan của người làm công tác phân loại ảnh. Quy trình phân loại ảnh dựa trên giải

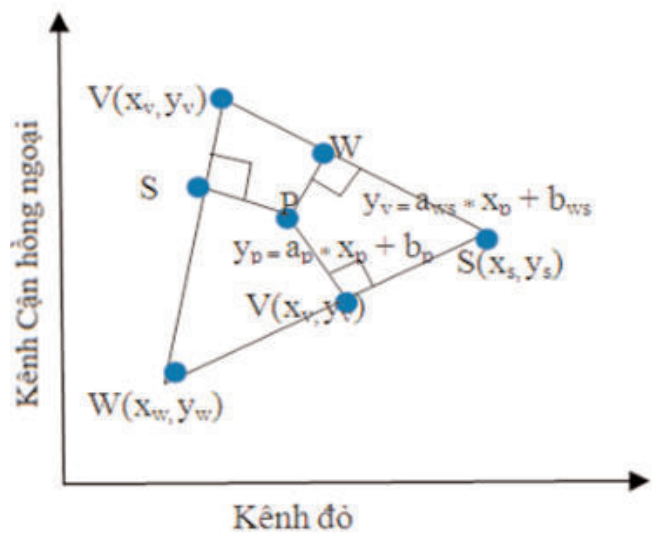

Hình 6: Tính toán tỷ lệ thành phần nước, đất, thực vật trong điểm ảnh 
pháp nâng cao độ chính xác của công tác phân loại ảnh dựa theo giá trị phổ và tỷ lệ thành phần đất, nước, thực vật, kết quả thực nghiệm, đánh giá độ chính xác của kết quả sẽ được trình bày trên số tới của Tạp chí Khoa học Đo đạc và Bản đồ. $\bigcirc$

\section{Tài liệu tham khảo}

[1]. Bateson, C. A., Asner, G. P., và Wessman, C. A. 2000. "Endmember bundles: A new approach to incorporating endmember variability into spectral mixture analysis". IEEE Trans. Geosci. Remote Sensing, Số. 38, trang. 1083-1094.

[2]. Bianchi. R., Cavalli. R., Fiumi. L. 2001. "CNR LARA project, Italy: Airborne laboratory for environmental research". Summaries of the V JPL Airborne Earth ScienceWorkshop, Pasadena, CA.

[3]. Baret, F., Guyot, G. 1991. Potentials and limit of vegetation indices for LAI and APAR assessment, Remote Sensing of Environment, Số 35, trang 161-173.

[4]. Bezdek, J. và Full, W. 1984. "FCM; The fuzzy c-means clustering algorithm", Computer and Geosciences, Số 10, trang 191-203.

[5]. Bosdogianni, P và Kittle, J. 1997. "Robus unmixing of large sets of mixed pixel", Pattern Recognition Letters, Số 18(5), trang 415-424

[6]. Boardman, J. W. và Kruse, F. A. 1994. "Automated spectral analysis: A geological example using AVIRIS data, Northern Grapevine Mountains, Nevada," in Proc. 10th Thematic Conference, Geologic Remote Sensing, San Antonio.

[7]. Boardman, W., Kruse, F. A. và Green, R. O. 1995. "Mapping target signatures via partial unmixing of AVIRIS data". Summaries of the V JPLAirborne Earth Science Workshop, Pasadena, CA.

[8]. Fisher, P. 1997. "The pixel: a snare and a delusion", International Journal of Remote Sensing, Số 18(3).

[9]. Foschi, G.P. 1994. A geometric approach to a mixed pixel problem: Detecting subpixel woody vegetation, Remote Sensing of Environment, Số 50(3), trang 317-327.

[10]. Jackson, R.D. 1983. Spectral Indices in n-Space, Remote Sensing of Environment, Số. 13, trang 409-421.

[11]. Floyd, F.S. 2013. Remote Sensing Principle and Interpretation.

[12]. Mao, C., Seal, M., và Heitschmidt, G. 1997. "Airborne hyperspectral image aquisition with digital CCD video camera". 16th Biennial Workshopon Videography and Color Photography in Resource Assessment, Weslaco, TX, trang 129-140.

[13]. Nageswara Rao, P.P. and Rao, V.R. 1987. Rice crop identification and area estimation using remotely-sensed data from Indian cropping patterns. International Journal of Remote Sensing, Số 8, trang 639-650.

[14]. Okamoto, K. and Fukuhara, M.1996. Estimation of paddy field area using the area ratio of categories in each mixel of Landsat TM. International Journal of Remote Sensing, Số 17, trang. 17351749 .

[15]. Qi, J., Chehbouni, A., Heute, A.R., Kerr, Y.H. 1994. Modified Soil Adjusted Vegetation Index (MSAVI), Remote Sensing of Environment, Số. 48, trang 119-126.

[16]. Petrou, M. và Foschi, P. G. 1999. "Confidence in linear spectral unmixing of single pixels". IEEE Trans. Geosci. Remote Sensing, Số. 37, trang. 624-626.

[17]. Richardson, A.J. and Wiegand, C.L. 1977. Distinguishing vegetation from soil background information, Photogrammetric 
Engineering and Remote Sensing, Số. 43, trang 1541-1552.

[18]. R. O. Green và ctv.1998. "Imaging spectroscopy and the airborne visible/infrared imaging spectrometer (AVIRIS)". Remote Sens. Environ., Số. 65, trang. 227-248, 1998.

[19]. Settle, J. 1996. "On the relationship between spectral unmixing and subspace projection". IEEE Trans. Geosci. Remote Sensing, Số. 34, trang.1045-1046.

[20]. Shimabukuro, Y.E và Smith, J.A. 1991. "The least squares unmixing models to generate fraction images derived from remote sensing multispectral data", IEEE Transactions on Geoscience and Remote Sensing, Số 29(1), trang 16-20.

[21]. Short, M.N. và ctv. The remote sensing tutorial. NASA/GSFC. http://rst.gsfc.nasa.gov.
[22]. Tadjudin. S. và Landgrebe. D., 1998. "Classification of high dimensional data with limited training samples". Ph.D. dissertation, School of Elect. Eng. Comput. Sci., Purdue Univ., Lafayette, IN.

[23]. Tennakoon, S.B., Murty, V.V.N., and Eiumnoh, A. 1992. Estimation of cropped area and grain yield of rice using remote sensing data. International Journal of Remote Sensing, Số 13, trang.427-439.

[24]. Tuekey, C.J, 1979. Red and photographic infrared linear combination for monitoring vegetation. Remote Sensing, Số 8, trang 127-15.

[25]. Yamagata, Y., Wiegand, C., Akiyama, T., Shibayama, M. 1988. Water turbidity and perpendicular vegetation indices for paddy rice flood damage analyses, Remote Sensing of Environment Environment, Số. 26, Trang 241-251.O

\section{Summary}

\section{The development of an approach for enhancing satellite image classification} accuracy applied to mixed land surface - Methodology Section

\section{Dr. Pham Minh Hai, Institute of Geodesy and Cartography}

Satellites imagery applications nowadays, serving the purposes of research, experiment or social life in general and in the field of Environment in particular, are increasingly popular. The two commonly used processes of extracting information are unsupervised and supervised classification. Supervised classification is the process of clustering pixels into classes based on training data (groups of pixels that represent areas) that you define. In supervised classification, a user can select sample pixels in an image that are representative of specific classes and then direct the image processing software to use these training sites (testing sets or input classes) as references for the classification of all other pixels in the image. However, when performing supervised classification in the area with mixed land surface, the lower accuracy of image classification is attributed to the effect of mixed pixels. Mixed pixel is a phenomenon that occurs when pixels with values belonging to class $A$, but are classified in other classes instead of class A. In data applications using small and medium-resolution, surface patches imaged as an individual pixels may contain more than one cover-type. As a result, mixed pixel classification by handling some components, including soil, water, vegetation on each pixel to improve the accuracy of classification results, is an important issue. Considering the present situation, the authors carried out the theme "The development of an approach for enhancing satellite image classification accuracy applied to mixed land surface - Methodology Section". O 